\title{
Gauge principle revisited: towards a unification of space-time and internal gauge interactions $^{1}$
}

\author{
V. Aldaya ${ }^{2,3}$, J.L. Jaramillo ${ }^{2,3}$ and J. Guerrero ${ }^{2,3,4}$
}

\begin{abstract}
The minimal coupling principle is revisited under the quantum perspectives of the space-time symmetry. This revision is better realized on a Group Approach to Quantization (GAQ) where group cohomology and extensions of groups play a preponderant role. We firstly consider the case of the electromagnetic potential; the Galilei and/or Poincaré group is (non-centrally) extended by the "local" $U(1)$ group. This group can also be seen as a central extension, parametrized by both the mass and the electric charge, of an infinite-dimensional group, on which GAQ leads to the dynamics of a particle moving in the presence of an electromagnetic field. Then we try the gravitational interaction of a particle by making the spacetime translations "local". However, promoting to "local" the space-time subgroup of the true symmetry of the quantum free relativistic particle, i.e. the centrally extended by $U(1)$ Poincaré group, results in a new electromagnetic-like force of pure gravitational origin. This is a consequence of the space-time translations not being an invariant subgroup of the extended Poincaré group and constitutes a preliminary attempt to a non-trivial mixing of space-time and internal gauge interactions.
\end{abstract}

PACS: 02.40.-k, 03.65.Fd, 11.15.-q

\footnotetext{
${ }^{1}$ Work partially supported by the DGICYT.

${ }^{2}$ Instituto de Astrofísica de Andalucía, Apartado Postal 3004, Granada 18080, Spain

${ }^{3}$ Instituto de Física Teórica y Computacional Carlos I, Facultad de Ciencias, Universidad de Granada, Campus de Fuentenueva, Granada 18002, Spain

${ }^{4}$ Departamento de Matemática Aplicada, Facultad de Informática, Campus de Espinardo, 30100 Murcia, Spain
} 


\section{Introduction}

In the Lagrangian formalism, formulated on the 1-jet bundle $J^{1}(E)$ of a vector bundle $E$ on Minkowski space-time $M$, promoting a given underlying rigid symmetry to "local", i.e. extending the corresponding Lie algebra by taking the tensor product of it by the algebra of real analytic functions on $M$, requires the introduction of a derivation law on the module of sections of $E, \Gamma(E)$, which is eventually interpreted as a potential providing the corresponding gauge interaction. This is essentially the formulation of the so-called Minimal Coupling Principle, which culminates in Utiyama's theory [3]. Internal gauge invariance had originally led successfully to electromagnetic interaction associated with $U(1)$, then to Yang-Mills associated with isospin $S U(2)$ (valid only at the "very strong" limit), electroweak with $(S U(2) \otimes U(1)) / Z_{2}$, and finally to strong interaction associated with colour $S U(3)$. The same spirit is shared by later attempts to unify all of these into gauge groups such as $S U(5)$. On the other hand, the "local" invariance under external (space-time) symmetries, such as a subgroup of the Poincaré group, has been used to provide a gauge framework for gravity [4], although fully disconnected from the other (internal) interactions. In fact, a unification of gravity and the other interactions would have supposedly required the non-trivial mixing of the space-time group and some internal symmetry, a task explicitely forbidden by the so-called no-go theorems by O'Raifeartaigh, Coleman, Mandula, Michel, etc. [5, 6, 7, 8] long ago, which stated that there is no finite-dimensional Lie group containing the Poincaré group acting as diffeomorphisms of the base manifold $M$, the Minkowski space-time, and any internal $S U(n)$ group acting linearly on the fibre of $E$, except for the direct product. It is worth mentioning that supersymmetry was originally developped in the 70's, mainly by Salam and Strathdee [9], in an unsuccessful attempt to invalidate the no-go theorems.

However, the current skill in dealing with Lie group extensions and irreducible representations of (even infinite-dimensional) Lie groups tempts us into revisiting the question of the mixing of symmetries and, accordingly, the unification of interactions in terms of ordinary Lie groups. We propose a simple, yet non-trivial, way of facing the problem of interaction mixing. This consists in identifying one of the $U(1)$ Cartan subgroups in the internal symmetry with the $U(1)$ phase invariance in Quantum Mechanics. Then, turning the space-time translation subgroup of a centrally extended Poincaré group [10, 11, 12, 13] into a "local" group automatically promotes the original rigid internal symmetry to the gauge level in a non-trivial way from the physical standpoint. This provides a non-trivial mixing of gravity and the already introduced internal interaction associated with the given unitary symmetry. Here, we seek to demonstrate explicitly the ocurrence of this new phenomenon at least at a given approximation without exhausting all possibilities of the proposed algorithm. This means that our present computational outputs must be understood as being partial, although, at the non-relativistic limit, they are exact and reveal with precision the mixing effect [14].

As is wellknown, the Minimal Coupling Principle can also be formulated on a bundle $E$ of the form $E=\mathbb{R}^{3} \times \mathbb{R} \rightarrow \mathbb{R}$, as corresponding to the case of Mechanics, thus making the problem technically easier while keeping the essential point to be discussed here. In 
this framework, to be followed in the present paper, the gauge principle will be revisited by simply substituting the Quantum Mechanical space-time symmetry for the standard (classical) one. In fact, in a previous letter [14], we sketched an approach to the problem in this simple and economical way, i.e. in a Particle Mechanics (versus Field Theory) framework, and we now undertake a more detailed and formal presentation. A much more involved generalization to Quantum Field Theory is under way [15.

Since the revision we attempt here lies entirely on symmetry grounds, a (Quantum) Mechanical formulation tightly attached to a group structure is better suited. There is, in fact, a way of associating physical dynamics with a specific symmetry group. This could be accomplished by means of the rather standard co-adjoint-orbits method of Kirillov [16], where the Lagrangian is seen as the local potential of the corresponding symplectic form. However, we shall proceed through a group approach to quantization (GAQ) 17, 18] which is directly related to the co-homological structure of the symmetry group, and leads directly to the quantum theory, a fact that can be of capital relevance in the near future in passing to the quantum-field-theory level. Co-homology parameters will be directly identified with the physical coupling constants. In this sense, the association of the parameter of the (symplectic) co-homology group of the Galilei group with the particle mass has been emphasized by Souriau 19.

As an intermediate step between the more standard Lagrangian version of the Minimal Interaction Principle and the one to be presented here, we shall formulate a version "a la Cartan", i.e. in terms of the invariance of the Poincaré-Cartan form [20, 21, 22, 23] rather than the Lagrangian, of the part of Utiyama's theory concerning the particle in interaction with the field. Indeed, as mentioned above, the dynamics of the gauge fields themselves will be considerd elsewhere.

The paper is organized as follows. Sec. II is a thorough presentation of basic geometrical aspects of Classical and Quantum Mechanics, mainly those fundamental to the development of the present work. Sec. III is devoted to the Cartan-like analysis of the Minimal Coupling Principle particularized for the case of electromagnetism and non-relativistic gravity. In Sec. IV we present explicitly the GAQ with the example of a particle moving in an electromagnetic field. Finally, in Sec. V, we directly present the chief problem of gauging the translation subgroup of the centrally extended Poincaré group giving rise to the new phenomenon of an extra coupling constant mixing non-trivially the geodesic force and the Lorentz one. Some outlooks are included at the end.

\section{Phase invariance in Quantum Mechanics}

According to the standard approach to Quantum Mechanics (see for instance [24]) a state of the system is characterized by a ray, rather than a vector, of a Hilbert space i.e. normalized wave functions are determined up to a complex number of module 1 or phase. This is a direct consequence of the definition of probability and constitutes a symmetry

to be referred to as $U(1)$ or Phase Invariance in Quantum Mechanics. Let us approach this symmetry from quite different perspectives to highlight its fundamental features. 


\subsection{Behaviour of the Schrödinger equation}

We shall consider the behaviour of the Schrödinger equation corresponding to the free quantum particle

$$
i \hbar \frac{\partial}{\partial t} \Psi=-\frac{\hbar^{2}}{2 m} \nabla^{2} \Psi
$$

under the Galilei transformations:

$$
\begin{aligned}
t^{\prime} & =t+b \\
\vec{x}^{\prime} & =R \vec{x}+\vec{a}+\vec{V} t \\
\vec{v}^{\prime} & =R \vec{v}+\vec{V} .
\end{aligned}
$$

where $R$ represents rotations, $b \in \mathbb{R}$ and $\vec{a} \in \mathbb{R}^{3}$ time and space translations, respectively, and $\vec{V} \in \mathbb{R}^{3}$ galilean boosts.

Equation (11) acquires an extra term,

$$
i \hbar \frac{\partial}{\partial t^{\prime}} \Psi+i \hbar \vec{V} \cdot \frac{\partial \Psi}{\partial \vec{x}^{\prime}}=-\frac{\hbar^{2}}{2 m} \nabla^{\prime 2} \Psi
$$

which can be compensated only by also transforming the wave function. Allowing for a non-trivial phase factor in front of the transformed wave function, of the form

$$
\Psi^{\prime}=e^{\frac{i m}{\hbar}\left(\vec{V} \cdot R \vec{x}+\frac{1}{2} \vec{V}^{2} t\right)} \Psi
$$

the Schrödinger equation becomes strictly invariant:

$$
i \hbar \frac{\partial}{\partial t^{\prime}} \Psi^{\prime}=-\frac{\hbar^{2}}{2 m} \nabla^{\prime 2} \Psi^{\prime}
$$

The need for a transformation such as (1) accompanying the space-time transformation (2) to accomplish full invariance strongly suggests the adoption of a central extension of the Galilei group as the basic (quantum-mechanical) space-time symmetry for the free particle 25]. The constant $\hbar$ is required to keep the exponent in (4) dimensionless.

The successive composition of two transformations in the extended Galilei group $\tilde{G}$ immediately leads to the group law:

$$
\begin{aligned}
& b^{\prime \prime}=b^{\prime}+b \\
& \vec{a}^{\prime \prime}=\vec{a}^{\prime}+R\left(\overrightarrow{\epsilon^{\prime}}\right) \vec{a}+\vec{V}^{\prime} b \\
& \vec{V}^{\prime \prime}=\vec{V}^{\prime}+R\left(\overrightarrow{\epsilon^{\prime}}\right) \vec{V}
\end{aligned}
$$

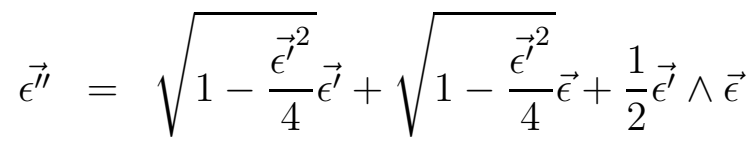

$$
\begin{aligned}
& e^{i \phi^{\prime \prime}}=e^{i \phi^{\prime}} e^{i \phi} e^{\frac{i m}{\hbar}\left[\vec{V}^{\prime} R^{\prime} \vec{a}+\frac{1}{2} b \vec{V}^{\prime 2}\right]},
\end{aligned}
$$

where $e^{i \phi} \in U(1)$, and we have made the rotation parameters $\vec{\epsilon} \in \mathbb{R}^{3}$ explicit, which are restricted to $2 \sin \frac{\chi}{2}=|\vec{\epsilon}|, \chi$ being the rotation angle. 


\subsection{Semi-invariance in Classical Mechanics}

This phenomenon of extending the space-time symmetry, although conceptually pure quantum mechanics, can also be recast within a (semi-)classical formalism, by requiring the simultaneous extension of the classical phase space by a new variable $\phi$, transforming in a non-trivial way under the $U(1)$-extended symmetry group. The need for such an extension is motivated by the lack of strict invariance of the Poincaré-Cartan form associated with the free particle $\left(H=p_{i} \dot{x}^{i}-L=\frac{\vec{p}^{2}}{2 m}\right)$,

$$
\Theta_{P C} \equiv p_{i} d x^{i}-H d t=\left(\frac{\partial L}{\partial \dot{x}^{i}}\left(d x^{i}-\dot{x}^{i} d t\right)+L d t\right)=p_{i} d x^{i}-\frac{\vec{p}^{2}}{2 m} d t
$$

under the Galilei group. In fact, it is left only semi-invariant by the infinitesimal transformations associated with (2) in the sense that the Lie derivative of $\Theta_{P C}$ with respect to those generators is the differential of a function not necessarily zero:

$$
\begin{aligned}
& X_{b}=\frac{\partial}{\partial t} \quad \Rightarrow \quad L_{X_{b}} \Theta_{P C}=0 \\
& X_{\vec{a}}=\frac{\partial}{\partial \vec{x}} \quad \Rightarrow \quad L_{X_{\vec{a}}} \Theta_{P C}=0 \\
& X_{\vec{V}}=t \frac{\partial}{\partial \vec{x}}+m \frac{\partial}{\partial \vec{p}} \quad \Rightarrow \quad L_{X_{\vec{V}}} \Theta_{P C}=d(m \vec{x}) \\
& X_{\vec{\epsilon}}=\vec{x} \wedge \frac{\partial}{\partial \vec{x}}+\vec{p} \wedge \frac{\partial}{\partial \vec{p}} \quad \Rightarrow \quad L_{X_{\vec{\epsilon}}} \Theta_{P C}=0
\end{aligned}
$$

The pathology of semi-invariance is parallel to the absence of a clean quotient by the equations of motion. Let us see in some detail the quotient process in going to the solution manifold. In the Cartan formalism the trajectories of a general physical system are the orbits of the kernel of $d \Theta_{P C}$ :

$$
\begin{aligned}
\Theta_{P C} & \equiv p_{i} d x^{i}-H d t \\
\Omega & \equiv d \Theta_{P C}=d p_{i} \wedge d x^{i}-\frac{\partial H}{\partial x^{i}} d x^{i} \wedge d t-\frac{\partial H}{\partial p_{i}} d p_{i} \wedge d t .
\end{aligned}
$$

$\Omega$ has a one-dimensional kernel generated by $X^{H} \in \operatorname{Ker} d \Theta_{P C}$ such that $d t\left(X^{H}\right)=1$,

$$
X^{H}=\frac{\partial}{\partial t}+\frac{\partial H}{\partial p_{i}} \frac{\partial}{\partial x^{i}}-\frac{\partial H}{\partial x^{i}} \frac{\partial}{\partial p_{i}},
$$

and the associated equations of motion are the Hamilton equations:

$$
\begin{aligned}
\frac{d t}{d \tau} & =1 \\
\frac{d x^{i}}{d \tau} & =\frac{\partial H}{\partial p_{i}} \\
\frac{d p_{i}}{d \tau} & =-\frac{\partial H}{\partial x^{i}}
\end{aligned}
$$

The vector field $X^{H}$ defines a one-parameter group which divides the space of movements $\mathbb{R} \times \mathbb{R}^{3} \times \mathbb{R}^{3}$, parametrized by $(t, \vec{x}, \vec{p})$, into classes, and $M \equiv\left\{\mathbb{R} \times \mathbb{R}^{3} \times \mathbb{R}^{3}\right\} / X^{H}$ 
constitutes the symplectic phase space of the system characterized by the Hamiltonian $H$; the symplectic form is obtained by the projection of $\Omega$. The change of variables under which the equations of motion on the quotient become trivial is the Hamilton-Jacobi transformation. For the example $H=\frac{\vec{p}^{2}}{2 m}$, corresponding to a free particle, this transformation is:

$$
\left\{\begin{array} { l } 
{ x ^ { i } = \frac { P ^ { i } } { m } \tau + K ^ { i } } \\
{ p _ { i } = P _ { i } } \\
{ t = \tau }
\end{array} \Longleftrightarrow \left\{\begin{array}{l}
K^{i}=x^{i}-\frac{p^{i}}{m} t \\
P_{i}=p_{i} \\
\tau=t
\end{array}\right.\right.
$$

where the constants of motion $K^{i}, P_{j}$ parametrize the solution manifold $M$. However, the form $\Theta_{P C}$ goes to the quotient except for a total differential:

$$
\begin{aligned}
\Theta_{P C} & \rightarrow P_{i} d K^{i}+d\left(\frac{\vec{P}^{2}}{2 m} \tau\right) \\
\omega & =d P_{i} \wedge d K^{i}
\end{aligned}
$$

\subsection{Poisson algebra realization}

Another equivalent Analytical-Mechanics breakdown claiming a "generalization" is the unfair relationship between the Lie brackets of basic symmetries and the corresponding Poisson brackets of the associated Noether invariants. The symplectic form [21, 22, 23] is a skew-symmetric "metric" and defines an isomorphism $\omega^{b}: \mathcal{X}(M) \leftrightarrow \Lambda^{1}(M)$ between the vector space of vector fields on $M$ and that of one-forms on $M$,

$$
X \in \mathcal{X}(M) \longmapsto \omega(X, \cdot) \equiv i_{X} \omega \in \Lambda^{1}(M),
$$

associating a bracket $\{$,$\} on \Lambda^{1}(M)$ with the Lie bracket of vector fields. In particular, given functions $f, g \in C^{\infty}(M)$, their differentials are associated with Hamiltonian vector fields $X_{f}, X_{g}$ [26]. This permits the definition of a Poisson bracket between functions, rather than one-forms, but this time the correspondence $\{,\} \rightarrow[$,$] ,$

$$
\{,\}: f, g \longmapsto\{f, g\} / d\{f, g\}=-i_{\left[X_{f}, X_{g}\right]} \omega,
$$

is no longer an isomorphism because constant functions have trivial Hamiltonian vector

fields. In particular, with regard to the example $H=\frac{\vec{p}^{2}}{2 m}$, and considering $K^{i}, P_{j}$ as the basic coordinates for $M$, we find:

$$
\left\{K^{i}, P_{j}\right\}=\delta_{j}^{i} \cdot 1 \longmapsto\left[X_{K^{i}}, X_{P_{j}}\right]=0
$$

that is, a Lie algebra homomorphism whose kernel is the central subalgebra of constant functions, $\mathbb{R}$, generated by 1 . It is easy to realize that $X_{K^{i}}, X_{P_{j}}$ are nothing other than 
the generators $X_{V^{i}}, X_{a^{j}}$, respectively, of the action (2) of the unextended Galilei group on the space of movements, written on the solution manifold [27].

The extension of phase space is required to represent faithfully the (classical) Poisson algebra by means of the generators of the extended symmetry as first-order differential operators, a fact that constitutes the Bohr-Sommerfeld approximation to Quantization or Prequantization, in the language of Geometric Quantization [19, 28, 29, 30]. Let us look at the preliminary steps towards the geometric attempts at quantization.

\subsection{Geometric and group approach to quantization}

The existence of a non-trivial kernel in the correspondence between functions and Hamiltonian vector fields is an essential failure to the naive geometric approach to quantization ^: $f \mapsto \hat{f} \equiv X_{f}$, which would associate the trivial operator to any constant. The simplest way of avoiding this problem consists of enlarging phase space (and/or movements space) with one extra variable providing one extra component to $X_{f}$, and generalizing accordingly the equation $i_{X_{f}} d \Theta_{P C}=-d f$ so as to get a non-trivial new component even though $f$ is a constant. On a quantum manifold $P$, locally isomorphic to $M \times S^{1}$, with connection form $\Theta$ such that the curvature two-form $(d \Theta)$ coincides with $d \Theta_{P C}$, the equation above can be replaced by the set of equations [19]:

$$
\begin{aligned}
i_{\tilde{X}_{f}} d \Theta & =-d f \\
i_{\tilde{X}_{f}} \Theta & =f
\end{aligned}
$$

generalizing this way the quantization map which now reads (except perhaps for a minus sign)

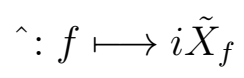

Note that Eq. (17) inmediately implies the strict invariance of $\Theta$ under $\tilde{X}_{f}$ :

$$
L_{\tilde{X}_{f}} \Theta=d i_{\tilde{X}_{f}} \Theta+i_{\tilde{X}_{f}} d \Theta=d f-d f=0
$$

Locally, we can write $\Theta=\Theta_{P C}+\frac{d z}{i z}, z=e^{i \Phi} \in S^{1}$ and then $\tilde{X}_{f}=X_{f}+[f-$ $\left.\Theta_{P C}\left(X_{f}\right)\right]\left(i z \frac{\partial}{\partial z}-i z^{*} \frac{\partial}{\partial z^{*}}\right)$, and we immediately see that (17) has a unique solution associating the fundamental (vertical) vector field $\Xi \equiv i z \frac{\partial}{\partial z}-i z^{*} \frac{\partial}{\partial z^{*}}=\frac{\partial}{\partial \Phi}$, dual to $\frac{d z}{i z}$, with the unity of $\mathbb{R}$.

The quantization map ^ is now an isomorphism between the Poisson algebra on $M$ and the Lie subalgebra of vector fields on $P$ that are solutions to (17). For the basic functions, we have:

$$
\left\{K^{i}, P_{j}\right\}=\delta_{j}^{i} \cdot 1 \longleftrightarrow\left[\tilde{X}_{K^{i}}, \tilde{X}_{P_{j}}\right]=\delta_{j}^{i} \cdot \Xi
$$

It is again easy to realize that, in the case of the free particle, the operators $\tilde{X}_{K^{i}}, \tilde{X}_{P_{j}}$ are nothing other than the generators $\tilde{X}_{V^{i}}, \tilde{X}_{a^{j}}$ of the action (2) and (幽) on the extended 
space of movements (with $\zeta \equiv e^{i \phi} \in S^{1}$ ) of the extended Galilei group, that is to say,

$$
\begin{aligned}
\tilde{X}_{b} & =X_{b} \\
\tilde{X}_{\vec{a}} & =X_{\vec{a}} \\
\tilde{X}_{\vec{V}} & =X_{\vec{V}}-\frac{1}{\hbar} m \vec{x} \frac{\partial}{\partial \phi} \\
\tilde{X}_{\vec{\epsilon}} & =X_{\vec{\epsilon}}
\end{aligned}
$$

written on the solution manifold.

To pass to the solution manifold, we must take into account the evolution of the new variable $\zeta \in U(1)$. In fact, the equations of motion in the extended (by $U(1)$ ) movement space are given by the vector field $\check{X}$ in the kernel of $d \Theta$ and $\Theta$, simultaneously, satisfying $d t(X)=1$. Locally, and for the choice $\Theta_{P C}=p_{i} d x^{i}-H d t$ we find:

$$
\check{X}^{H}=X^{H}+\left\{H-p_{i} \frac{\partial H}{\partial p_{i}}\right\} \Xi
$$

For the free particle, $\check{X}^{H}$ provides the following new equation, to be added to de HamiltonJacobi set (12),

$$
\zeta=z e^{-\frac{i}{\hbar} \frac{\vec{P}^{2}}{2 m} t} \Longleftrightarrow z=\zeta e^{\frac{i}{\hbar} \frac{\vec{p}^{2}}{2 m} t}
$$

Now the form $\Theta$, originally written in the space of movements as

$$
\Theta=p_{i} d x^{i}-\frac{\vec{p}^{2}}{2 m} d t+\hbar \frac{d \zeta}{i \zeta}
$$

goes to the quotient, by applying the extended Hamilton-Jacobi transformation (12) and (23), giving

$$
\Theta=P_{i} d K^{i}+\hbar \frac{d z}{i z}
$$

The space of wave functions $\Psi$ is constituted by the complex functions on $P$ that satisfy the $U(1)$-equivariance condition, turning $\Psi$ into a section of the principal bundle $P \rightarrow M[31]:$

$$
\Sigma \tilde{\Psi}=i \tilde{\Psi} \longleftrightarrow \tilde{\Psi}(K, P, z)=z \Psi(K, P)
$$

on which the vector fields $\tilde{X}_{f}$ act, defining the pre-quantum operators.

Unfortunately, the quantization map ${ }^{\wedge}$ is faithful but not irreducible as a representation of the Lie algebra of classical functions. At this prequantization level, we are able to reproduce only the Bohr-Sommerfeld-Wilson quantization rules [30]. We know that this representation is reducible because of the existence of non-trivial operators commuting with the basic quantum generators $\hat{K}^{i} \equiv i \hbar \tilde{X}_{K^{i}}, \hat{P}_{j} \equiv-i \hbar \tilde{X}_{P_{j}}$. In fact, thinking of the simplest case, that of the free particle for example, and adopting for $\Theta$ the local expression (25), we get the following basic operators acting on the untilded wave functions $\Psi$ : 


$$
\begin{aligned}
\hat{K}^{i} & =i \hbar \frac{\partial}{\partial P_{i}}+K^{i} \\
\hat{P}_{j} & =-i \hbar \frac{\partial}{\partial K^{j}}
\end{aligned}
$$

and it is clear that the operators $\check{K}^{i} \equiv \frac{\partial}{\partial P_{i}}$ do commute with them.

True quantization requires that all non-trivial operators commuting with basic quantum generators should be trivialized. We must then impose a maximal set of mutually compatible conditions in the form $X \Psi=0$, for $X$ in some maximal vector space called polarization. For instance, in the example above the operator $\frac{\partial}{\partial P_{i}}$ would be trivial had we imposed the polarization condition $\frac{\partial}{\partial P_{i}} \Psi=0 \rightarrow \Psi \neq \Psi(P)$. Finding a polarization, however, is a non-trivial task in general, because two polarization conditions $\hat{a} \Psi=0, \hat{b} \Psi=0$ are inconsistent if $[\hat{a}, \hat{b}]=\hat{1}$ and once a certain polarization has been imposed, the set of physical operators that preserve the polarization is severely restricted. Even more, the existence of an invariant polarization, i.e. a polarization preserved by the basic operators, is by no means guaranteed.

A stylish and even practical (at least for fundamental systems) solution to this and other problems comes from the structure itself of the (classical) Poisson algebra seen as a fundamental symmetry of the physical system to be quantized or, more precisely, the (quantum) physical system to be studied. Looking again at the quantum symmetry of the free particle, the extended Galilei group (2), we can adopt its structure as the basic block to provide the whole physical system, including the space and time where the evolution takes place [17, 18]. The chief idea is to replace the quantum manifold $P$ with a Lie group $\tilde{G}$ bearing the structure of a principal bundle with structure group $U(1)$ and a connection 1-form $\Theta$ selected from the canonical invariant forms on the group. The simplest, though sufficiently broad, example of such a class of Lie groups is the case of a central extension of a given group $G$ by $U(1)$ 25]. In this situation, the central extensions are parametrized by the second co-homology group of $G$ in $U(1), H^{2}(G, U(1))$, and the coordinates in this (vector) space are associated with fundamental constants such as the mass [19] or the electric charge (see Sec. IV). Each co-homology constant is in turn associated with a Lie subalgebra of even dimension (in fact, a symplectic vector space) which will provide a set of canonically conjugate pairs of operators. We shall call them dynamical quantities, or the corresponding parameters in the group (classical) dynamical variables.

The virtues of working on a Lie group are multiple, but, for the time being, let us point out that of possessing two sets of natural, mutually commuting operators; that is, the leftand right-invariant vector fields, the latter of which can provide a unitary representation to be reduced by polarization conditions imposed by a subalgebra of the former. With this choice, the connection 1 -form $\Theta$ is the $U(1)$ component of the left-invariant canonical 1 -form on the group, which is automatically invariant under the right-invariant vector fields. The only apparent drawback of doing so is that the quotient $\tilde{G} / U(1)$ is not necessarily a symplectic manifold since the curvature 2-form $d \Theta$ may have a non-trivial kernel. 
However, this apparent problem is solved by including in the polarization conditions, formulated in terms of a maximal horizontal subalgebra $\mathcal{P}$ of left-invariant vector fields, the subalgebra of (left-invariant) vector fields $\mathcal{G}_{\Theta}$ generating the characteristic module of $\Theta$, i.e. $\operatorname{Ker} \Theta \cap \operatorname{Ker} d \Theta$.

It should be stressed that, far from being a drawback, working on the pre-contact manifold $\tilde{G}$ instead of a proper quantum manifold $P$ allows us to deal with quantum systems without classical limit. In fact, the trajectories of the vector fields in the characteristic subalgebra generalize the classical motion, and the solution of the corresponding equations can be bypassed by including this subalgebra in the Polarization as generalized Schrödinger equations.

\section{Cartan-like version of the Minimal-Coupling Prin- ciple: the electromagnetic and non-relativistic grav- itational forces}

Once the symmetry of the free particle has been posed through the strict invariance of the corresponding extended Poincaré-Cartan (or quantization) form $\Theta$ (24) under the action of extended Galilei group, we may postulate the requirement of invariance of a generalized $\Theta$ under the Galilei group (non-centrally) extended by the "local" group $U(1)(\vec{x}, t)$ (of local phase transformations $\left.e^{i \phi(\vec{x}, t)}\right)$. This requirement, along with the minimal substitution in $\Theta$ to achieve strict invariance, constitutes the Cartan-like version of the Minimal Coupling Principle for the $U(1)$ rigid symmetry and will lead to the motion of a particle in the presence of an electromagnetic field.

Let us consider the Lie algebra $\tilde{\mathcal{G}}$ of the centrally extended Galilei group $\tilde{G}$ (only non-zero commutators):

$$
\begin{array}{rlrl}
{\left[\tilde{X}_{V^{i}}, \tilde{X}_{b}\right]} & =\tilde{X}_{a^{i}} & {\left[\tilde{X}_{V^{i}}, \tilde{X}_{a^{j}}\right]} & =\frac{m}{\hbar} \delta_{i j} \tilde{X}_{\phi} \\
& \left.\tilde{X}_{\epsilon^{i}}, \tilde{X}_{\epsilon^{j}}\right]=\epsilon_{i j .}{ }^{k} \tilde{X}_{\epsilon^{k}} & {\left[\tilde{X}_{\epsilon^{i}}, \tilde{X}_{V^{j}}\right]=\epsilon_{i j}{ }^{k} \tilde{X}_{V^{k}} \quad\left[\tilde{X}_{\epsilon^{i}}, \tilde{X}_{a^{j}}\right]=\epsilon_{i j}{ }^{k} \tilde{X}_{a^{k}}}
\end{array}
$$

which, as mentioned above, leaves strictly invariant the extended Poincaré-Cartan form $\Theta=p_{i} d x^{i}-\frac{\vec{p}^{2}}{2 m}+\hbar d \phi$, that is, $L_{\tilde{X}_{a}} \Theta=0, \forall \tilde{X}_{a} \in \tilde{\mathcal{G}}$.

Local $U(1)$ transformations generated by $f \otimes X_{\phi}, f$ being a real function $f(\vec{x}, t)$, are incorporated into the scheme by adding to (28) the extra commutators [32]:

$$
\left[\tilde{X}_{a}, f \otimes X_{\phi}\right]=\left(L_{\tilde{X}_{a}} f\right) \otimes X_{\phi}
$$

The Lie derivative of (24) with respect to $f \otimes X_{\phi}$ gives:

$$
L_{f \otimes X_{\phi}} \Theta=d\left(i_{f X_{\phi}} \Theta\right)+i_{f X_{\phi}} d \Theta=d f
$$

Keeping the strict invariance requires modifying $\Theta$ by adding a connection term $\Gamma=$ $\Gamma_{i} d x^{i}+\Gamma_{0} d t$ whose components transform under $U(1)(\vec{x}, t)$ as the space-time gradient 
of the function $f[33]$. Additional conditions on $\Gamma$ will be obtained by requiring strict invariance of $\Theta^{\prime} \equiv \Theta+\Gamma$ under the complete group. The generators of the action of the whole group on the variables $\left(t, \vec{x}, \vec{p}, \vec{\Gamma}, \Gamma_{0}, \phi\right)$ are:

$$
\begin{aligned}
\tilde{X}_{b} & =\frac{\partial}{\partial t} \\
\tilde{X}_{\vec{a}} & =\frac{\partial}{\partial \vec{x}} \\
\tilde{X}_{\vec{V}} & =t \frac{\partial}{\partial \vec{x}}+m \frac{\partial}{\partial \vec{p}}+\vec{\Gamma} \frac{\partial}{\partial \Gamma_{0}}+\frac{m}{\hbar} \vec{x} \frac{\partial}{\partial \phi} \\
\tilde{X}_{\vec{\epsilon}} & =\vec{x} \wedge \frac{\partial}{\partial \vec{x}}+\vec{p} \wedge \frac{\partial}{\partial \vec{p}}+\vec{\Gamma} \wedge \frac{\partial}{\partial \vec{\Gamma}} \\
f \otimes X_{\phi} & =-\vec{\nabla} f \frac{\partial}{\partial \vec{\Gamma}}+\frac{\partial f}{\partial t} \frac{\partial}{\partial \Gamma_{0}}-\frac{f}{\hbar} \frac{\partial}{\partial \phi}
\end{aligned}
$$

Then, the infinitesimal conditions

$$
L_{X}\left(\Theta^{\prime}\right)=0
$$

implies the following finite transformation properties of the components of $\Gamma$ :

$$
\begin{aligned}
\vec{\Gamma}^{\prime} & =R \vec{\Gamma} \\
\Gamma_{0}^{\prime} & =\Gamma_{0}+\vec{V} \cdot R \vec{\Gamma}
\end{aligned}
$$

under a rotation and a boost, and

$$
\begin{aligned}
\vec{\Gamma}^{\prime} & =\vec{\Gamma}+\vec{\nabla} f \\
\Gamma_{0}^{\prime} & =\Gamma_{0}+\frac{\partial f}{\partial t}
\end{aligned}
$$

under an element of $U(1)(\vec{x}, t)$.

Let us now compute the simultaneous kernel of $\Theta^{\prime}$ and $d \Theta^{\prime}$ and write the equations of motion, rewriting the connection $\Gamma$ as $\Gamma \equiv q A_{i} d x^{i}-q A_{0} d t$. We then have (omitting the accent over $\Theta^{\prime}$ )

$$
\begin{gathered}
\Theta=m \vec{v} \cdot d \vec{x}-\frac{1}{2} m \vec{v}^{2} d t+q \vec{A} \cdot d \vec{x}-q A^{0} d t+\hbar d \phi \\
\check{X}=\frac{\partial}{\partial t}+\vec{v} \cdot \frac{\partial}{\partial \vec{x}}+\frac{q}{m}\left[\left(\frac{\partial A_{j}}{\partial x^{i}}-\frac{\partial A_{i}}{\partial x^{j}}\right) v^{j}-\frac{\partial A^{0}}{\partial x^{i}}-\frac{\partial A_{i}}{\partial t}\right] \frac{\partial}{\partial v_{i}}-\frac{1}{\hbar}\left[\frac{1}{2} m \vec{v}^{2}+q\left(\vec{v} \cdot \vec{A}-A^{0}\right)\right] \frac{\partial}{\partial \phi},
\end{gathered}
$$

It states the equations of motion of a charged particle of charge $q$ in an electromagnetic field:

$$
\begin{aligned}
\frac{d \vec{x}}{d t} & =\vec{v} \\
m \frac{d \vec{v}}{d t} & =q\left[\vec{v} \wedge(\vec{\nabla} \wedge \vec{A})-\vec{\nabla} A^{0}-\frac{\partial \vec{A}}{\partial t}\right] \\
\frac{d \phi}{d t} & =-\frac{1}{\hbar}\left(\frac{\vec{p}^{2}}{2 m}-\frac{q}{m} \vec{A} \cdot \vec{p}\right)
\end{aligned}
$$


The Minimal-Coupling Principle can also be applied to the case of Newtonian Gravity by requiring the space and time translation parameters to depend on time. Unlike the electromagnetic gauge principle, which can be directly extended to the relativistic situation, relativistic gravity is much more involved and will be analysed in Sec. V, mixed with electromagnetism as our central task.

For brevity, let us consider the 1+1-dimensional case. Starting with the unextended Galilei group, we promote the space and time translations to local in the more economical way; that is, turning the corresponding group parameters into functions of time. The gauge algebra is then

$$
\begin{aligned}
{\left[f(t) \otimes X_{b}, X_{b}\right] } & =-\frac{\partial f}{\partial t} \otimes X_{b} \\
{\left[f(t) \otimes X_{b}, X_{a}\right] } & =0 \\
{\left[f(t) \otimes X_{b}, X_{V}\right] } & =f(t) \otimes X_{a}
\end{aligned}
$$

According to the minimal prescription, we introduce a conection $\Gamma \equiv h d t$ to be added to the free Poincaré-Cartan form, and an extra component in $\frac{\partial}{\partial h}$ to the Galilei generators for non-trivial realization of the current algebra (36). Then the semi-invariance of

$$
\Theta_{P C}^{\prime}=p d x-\frac{p^{2}}{2 m} d t+h d t
$$

under the current algebra (36) fixes the new components in $h$, so that the complete expression of the Lie algebra of the unextended Galilei group with local (depending only on time) space and time translation subgroup becomes:

$$
\begin{aligned}
f(t) \otimes X_{b} & =f \frac{\partial}{\partial t}+\left(\frac{p^{2}}{2 m}-h\right) \frac{d f}{d t} \frac{\partial}{\partial h} \\
f(t) \otimes X_{a} & =f \frac{\partial}{\partial x}-p \frac{d f}{d t} \frac{\partial}{\partial h} \\
X_{V} & =t \frac{\partial}{\partial x}+m \frac{\partial}{\partial p} \\
f(t) \otimes X_{h} & =f \frac{\partial}{\partial h}
\end{aligned}
$$

where we have had to introduce the new local generator $f(t) \otimes X_{h}$ in order to close the current algebra. The new generator also leaves the form (37) semi-invariant. Strict invariance is now achieved by adding to generators $X$ new components in $\frac{\partial}{\partial \phi}$ with coefficients equal to $-g, g$ being such that $i_{X} d \Theta^{\prime}=d g$. This results in:

$$
\begin{aligned}
\widetilde{f(t) \otimes X_{b}} & =f \frac{\partial}{\partial t}+\left(\frac{p^{2}}{2 m}-h\right) \frac{d f}{d t} \frac{\partial}{\partial h}-\frac{f}{\hbar}\left(\frac{p^{2}}{2 m}-h\right) \frac{\partial}{\partial \phi} \\
\widetilde{f(t) \otimes X_{a}} & =f \frac{\partial}{\partial x}-p \frac{d f}{d t} \frac{\partial}{\partial h}+\frac{1}{\hbar} f p \frac{\partial}{\partial \phi} \\
\tilde{X}_{V} & =t \frac{\partial}{\partial x}+m \frac{\partial}{\partial p}-\frac{m}{\hbar}\left(x-\frac{p}{m} t\right) \frac{\partial}{\partial \phi} \\
\widetilde{f(t) \otimes X_{h}} & =f \frac{\partial}{\partial h}-\frac{f}{\hbar} \frac{\partial}{\partial \phi}
\end{aligned}
$$


The reason for $U(1)$-extending after gauging is clear: both processes definitely do not commute. Proceeding the other way round leads, precisely, to the new results of Sec. V.

The Cartan-like equations associated with (37) lead directly to the Newtonian gravity equations if we identify $h$ with the gravitational potential. This potential can be related to the component $g_{00}$ of a metric in the Newtonian limit of General Relativity via the expression $g_{00} \approx 1+h$.

\section{Group Approach to the Quantization of a particle moving in an electromagnetic field}

In this and the next section, we shall adopt the GAQ formalism as a generalization of the geometrical approach to Quantum Mechanics, although we shall be interested, for now, primarily in the classical equations of motion. As mentioned above, we seek to reproduce any dynamical or kinematical quantity or variable out of a Lie group so that notation such as $t, \vec{x}, \vec{p}$, etc. will refer to group variables (although directly identifiable with "physical" ones once the equations of motion are written).

Let us start by exponentiating the algebra (28) $+(29)$, originally performed on a given movement space, in order to arrive at an abstract Lie group from which to obtain all physical structures. This algebra is infinite-dimensional but, for real analytic functions $f$, the dynamical-variable content of it, in the sense of Sec. 2.4, is addressed by the (cohomological) structure of the finite-dimensional subalgebra generated by $\tilde{\mathcal{G}}$ along with those generators $f \otimes X_{\phi}$ with only linear functions, $t \otimes X_{\phi}$ and $x^{i} \otimes X_{\phi}$, to be called $X_{A^{0}}$ and $X_{A^{i}}$, respectively. The rest of functions contribute only to the characteristic (nondynamical) subalgebra and can be decoupled from the theory. Let us call $\tilde{G}_{E}$ this finitedimensional group. It proves to be enough to describe the dynamics of a particle moving in an electromagnetic field if we resort to the trick (see below) of assuming an explicit dependence $A^{\mu}=A^{\mu}(\vec{x}, t)$ once the 1-form $\Theta$ will be found. (This procedure is suggested by the possibility of writing an analytic function in the form $f(\vec{x}, t)=\phi+A_{\mu}(\vec{x}, t) x^{\mu}$, where $\phi=f(\overrightarrow{0}, 0)$.) We shall not be involved here with the corresponding quantum field theory.

The group $\tilde{G}_{E}$ can be given the following group law which extends that of the Galilei group (with parameters $t, \vec{x}, \vec{v} \equiv \frac{\vec{p}}{m}$ instead of $b, \vec{a}, \vec{V}$, respectively) and agree with the finite transformation (31) and (32):

$$
\begin{aligned}
t^{\prime \prime} & =t^{\prime}+t \\
\vec{x}^{\prime \prime} & =\vec{x}^{\prime}+R^{\prime} \vec{x}+\vec{v}^{\prime} t \\
\vec{v}^{\prime \prime} & =\vec{v}^{\prime}+R^{\prime} \vec{v} \\
R^{\prime \prime} & =R^{\prime} R \\
\vec{A}^{\prime \prime} & =\vec{A}^{\prime}+R^{\prime} \vec{A} \\
A_{0}^{\prime \prime} & =A_{0}^{\prime}+A_{0}+\vec{v}^{\prime} \cdot R^{\prime} \vec{A}
\end{aligned}
$$




$$
\begin{aligned}
\zeta^{\prime \prime} & =\zeta^{\prime} \zeta e^{i \xi_{m}\left(g^{\prime}, g\right)} e^{i \xi_{q}\left(g^{\prime}, g\right)} \\
\xi_{m}\left(g^{\prime}, g\right) & \equiv-\frac{m}{\hbar}\left[\overrightarrow{v^{\prime}} \cdot R^{\prime} \vec{x}+\frac{1}{2} t{\overrightarrow{v^{\prime}}}^{2}\right] \\
\xi_{q}\left(g^{\prime}, g\right) & \equiv-\frac{q}{\hbar}\left[\overrightarrow{A^{\prime}} \cdot R^{\prime} \vec{x}+t\left(\overrightarrow{v^{\prime}} \cdot \overrightarrow{A^{\prime}}-A_{0}^{\prime}\right)\right]
\end{aligned}
$$

where $\xi_{m}\left(g^{\prime}, g\right)$ is a standard Bargmann-like cocycle associated with the Galilei (sub)group, in particular with the symplectic submanifold of coordinates $\left(x^{i}, v_{j}\right)$, and $\xi_{q}\left(g^{\prime}, g\right)$ is a new cocycle, parametrized by the electric charge, as we shall see, associated with the symplectic submanifold of coordinates $\left(x^{i}, A_{j}\right)$. Both satisfy the cocycle conditions:

$$
\begin{aligned}
\xi\left(g^{\prime}, g\right)+\xi\left(g^{\prime} * g, g^{\prime \prime}\right) & =\xi\left(g^{\prime}, g * g^{\prime \prime}\right)+\xi\left(g, g^{\prime \prime}\right) \\
\xi(0, g)=\xi\left(g^{\prime}, 0\right) & =0
\end{aligned}
$$

intended to mantain the structure of group law after the extension. Since both cocycles are associated with intersecting symplectic submanifold ( $\vec{x}$ is in both) we should expect a mixed momentum variable conjugated to $\vec{x}$, to be identified with the minimally coupled momentum. This is the essence of Minimal Coupling in GAQ.

¿From (40) we derive left- and right-invariant vector fields and from the former the $\zeta$-component of the left-invariant canonical 1-form:

$$
\begin{aligned}
& \tilde{X}_{t}^{L}=\frac{\partial}{\partial t}+\vec{v} \cdot \frac{\partial}{\partial \vec{x}}-\frac{1}{\hbar}\left[\frac{1}{2} m \vec{v}^{2}+q\left(\vec{v} \cdot \vec{A}-A_{0}\right)\right] \Xi \\
& \tilde{X}_{\vec{x}}^{L}=R\left(\frac{\partial}{\partial \vec{x}}-\frac{1}{\hbar}[m \vec{v}+q \vec{A}] \Xi\right) \\
& \tilde{X}_{\vec{v}}^{L}=R\left(\frac{\partial}{\partial \vec{v}}\right) \\
& \tilde{X}_{\vec{\epsilon}}^{L}=\sqrt{1-\frac{\vec{\epsilon}^{2}}{4}} \frac{\partial}{\partial \vec{\epsilon}}-\frac{1}{2} \vec{\epsilon} \wedge \frac{\partial}{\partial \vec{\epsilon}} \\
& \tilde{X}_{\vec{A}}^{L}=R\left(\frac{\partial}{\partial \vec{A}}+\vec{v} \frac{\partial}{\partial \overrightarrow{A_{0}}}\right) \\
& \tilde{X}_{A_{0}}^{L}=\frac{\partial}{\partial A_{0}} \\
& \tilde{X}_{\zeta}^{L}=i\left(\zeta \frac{\partial}{\partial \zeta}-\zeta^{*} \frac{\partial}{\partial \zeta^{*}}\right) \equiv \frac{\partial}{\partial \phi} \equiv \Xi \\
& \tilde{X}_{t}^{R}=\frac{\partial}{\partial t} \\
& \tilde{X}_{\vec{x}}^{R}=\frac{\partial}{\partial \vec{x}} \\
& \tilde{X}_{\vec{v}}^{R}=\frac{\partial}{\partial \vec{v}}+t \frac{\partial}{\partial \vec{x}}
\end{aligned}
$$




$$
\begin{aligned}
& \tilde{X}_{\vec{\epsilon}}^{R}=\sqrt{1-\frac{\vec{\epsilon}^{2}}{4}} \frac{\partial}{\partial \vec{\epsilon}}+\frac{1}{2} \vec{\epsilon} \wedge \frac{\partial}{\partial \vec{\epsilon}} \\
& \tilde{X}_{\vec{A}}^{R}=\frac{\partial}{\partial \vec{A}}-q \vec{x} \Xi \\
& \tilde{X}_{A_{0}}^{R}=\frac{\partial}{\partial A_{0}}+q t \Xi \\
& \tilde{X}_{\zeta}^{R}=i\left(\zeta \frac{\partial}{\partial \zeta}-\zeta^{*} \frac{\partial}{\partial \zeta^{*}}\right) \equiv \frac{\partial}{\partial \phi} \equiv \Xi \\
& \Theta \equiv \hbar \theta^{(\zeta) L}=m \vec{v} \cdot d \vec{x}-\frac{1}{2} m \vec{v}^{2} d t+q \vec{A} \cdot d \vec{x}-q A_{0} d t+\hbar \frac{d \zeta}{i \zeta}
\end{aligned}
$$

The commutation relations of (let us say) left generators (omitting rotations, which operate in the standard way) are:

$$
\begin{aligned}
{\left[\tilde{X}_{t}^{L}, \tilde{X}_{x^{i}}^{L}\right]=0 } & {\left[\tilde{X}_{t}^{L}, \tilde{X}_{v^{i}}^{L}\right]=-\tilde{X}_{x^{i}}^{L} } & {\left[\tilde{X}_{x^{i}}^{L}, \tilde{X}_{v^{j}}^{L}\right]=\frac{m}{\hbar} \delta_{i j} \Xi } \\
{\left[\tilde{X}_{t}^{L}, \tilde{X}_{A^{i}}^{L}\right]=0 } & {\left[\tilde{X}_{t}^{L}, \tilde{X}_{A^{0}}^{L}\right]=-\frac{q}{\hbar} \Xi } & {\left[\tilde{X}_{x^{i}}^{L}, \tilde{X}_{A^{j}}^{L}\right]=\frac{q}{\hbar} \delta_{i j} \Xi } \\
{\left[\tilde{X}_{x^{i}}^{L}, \tilde{X}_{A^{0}}^{L}\right]=0 } & {\left[\tilde{X}_{v^{i}}^{L}, \tilde{X}_{A^{i}}^{L}\right]=\delta_{i j} \tilde{X}_{A^{0}}^{L} } & {\left[\tilde{X}_{v^{i}}^{L}, \tilde{X}_{A^{0}}^{L}\right]=0 }
\end{aligned}
$$

If we compute the characteristic module of $\Theta$, i.e. $\operatorname{Ker} d \Theta \cap \operatorname{Ker} \Theta$ for $q=0$, as corresponding to the free particle, we find that it is generated by a left subalgebra

$$
\left.\mathcal{G}_{\Theta}\right|_{q=0}=<\tilde{X}_{t}^{L}, \tilde{X}_{\vec{\epsilon}}^{L}, \tilde{X}_{\vec{A}}^{L}, \tilde{X}_{A_{0}}^{L}>
$$

leading to the trajectories (12) and (23) for the dynamical (symplectic) variables $\vec{x}$ and $\vec{p}$, and additional ones for the kinematical (non-symplectic) variables $\vec{\epsilon}, \vec{A}, A_{0}$ which decouple from the theory. The quotient of $\tilde{G}_{E} / U(1)$ by the generalized equations of motion is a symplectic manifold (the solution manifold) of dimension $3+3$. However, for non-zero $q$, we have

$$
\mathcal{G}_{\Theta}=<\tilde{X}_{\vec{\epsilon}}^{L}, \tilde{X}_{\vec{A}}^{L}-\frac{q}{m} \tilde{X}_{\vec{v}}^{L}>,
$$

leading to a symplectic manifold of dimension $4+4$. We should note that no time evolution appears as an equation of motion. This is because the electromagnetic cocycle lends dynamical character to $t$ as conjugate to $A_{0}$. The only characteristic vector field, apart from $\tilde{X}_{\vec{\epsilon}}^{L}$, which again simply decouples the variables $\vec{\epsilon}$, is the one defining the Minimal Coupling. In fact, the Noether invariants $i_{\tilde{X}} \Theta$ are:

$$
\begin{aligned}
i_{\tilde{X}_{t}^{R}} \Theta & =-\left(\frac{1}{2} m \vec{v}^{2}+q A_{0}\right) \\
i_{\tilde{X}_{\vec{x}}^{R}} \Theta & =m \vec{v}+q \vec{A} \equiv \vec{P} \\
i_{\tilde{X}_{\vec{v}}^{R}} \Theta & =-m(\vec{x}-\vec{v} t)+q \overrightarrow{A t} \\
i_{\tilde{X}_{\vec{R}}^{R}} \Theta & =-q \vec{x} \\
i_{\tilde{X}_{\vec{x}}^{R}} \Theta & =q t
\end{aligned}
$$


reproducing, in particular the "canonical momentun" $\vec{P} \equiv m \vec{v}+q \vec{A}$.

Real dynamics appear when we impose the "constraint" $A_{0, i}=A_{0, i}(\vec{x}, t)$ on $\Theta$, whose characteristic module turns out now to be generated by $\check{X}$ in (34), thus reproducing the standard equations of motion. The trick of introducing this constraint after the form $\Theta$ associated with $\tilde{G}_{E}$ has been computed can in fact be justified in mathematical terms although at the price of introducing an explicit infinite parametrization of the field $A_{\mu}$ by means of, for instance, Fourier coefficients $a_{\mu}(\vec{k}), a_{\nu}^{*}(\vec{k})$, and very importantly, an extra space-time translation group associated with this field, let us say $\chi_{\mu}$. That is, the general space-time position on which the field lies, conceptually differs from the spacetime position of the particle. Under these conditions a cocycle of this infinite-dimensional group can be introduced, contributing the form $\Theta$ with the term $q \vec{A}(\vec{x}, t) \cdot d \vec{x}-q A_{0}(\vec{x}, t) d t$ where

$$
A_{\mu}(\vec{x}, t)=\int \frac{d^{3} \vec{k}}{2 k^{o}}\left\{a_{\mu}(\vec{k}) e^{-i k \cdot x}+a_{\mu}^{*}(\vec{k}) e^{i k \cdot x}\right\}
$$

That is, the electromagnetic field evaluated on the trajectories of the particle. Now, the abovementioned constraint proves to be as natural as stating that, on a trajectory, the particle sees the field $A_{\mu}$ evaluated on $x_{\mu}$ rather than on $\chi_{\mu}$. This precise construction, along with the (also infinite-dimensional) cocycle providing dynamical content to the field variables $a_{\mu}(\vec{k}), a_{\nu}^{*}(\vec{k})$ themselves, deserves a separate work, which is in course.

We should say to conclude this section that this study can be repeated with the centrally extended Poincaré group $\tilde{P}$ [10, 11] (see also [13]) by promoting to local the $U(1)$ transformations and considering the finite-dimensional subgroup $\tilde{P}_{E}$ analogous to $\tilde{G}_{E}$.

\section{$5 \quad$ Mixing the electromagnetic and gravitational forces}

Let us now consider the gravitational interaction from our group-theoretical viewpoint (we shall omit $\hbar$ in this section). To this end, we start directly with the centrally extended Poincaré group $\tilde{P}$ and see how the fact that the translation generators produce the central term under commutation with some other generators (boosts) plays a singular role in the relationship between local space-time translations and local $U(1)$ transformations. Symbolically denoting the generators of translations by $P, P_{0}$, those of boosts by $K$ and the central one by $\Xi$, we find:

$$
[K, f \otimes P] \simeq\left(L_{K} f\right) \otimes P+f \otimes\left(P_{0}+\Xi\right) .
$$

This means that turning the translations into local symmetry also entails the local nature of the $U(1)$ phase. We thus expect a non-trivial mixing of gravity and electromagnetism into an infinite-dimensional electro-gravitational group.

We shall follow steps identical to those given in the former example. In turning the parameters of space-time translations local, we replace $x^{\mu}$ with $x^{\mu}+h_{\mu \nu}\left(\vec{x}, x^{0}\right) x^{\nu}$, write a finite-dimensional algebra $\mathcal{P}_{E G}$ keeping only the linear part of local space-time translations 
$\left(x^{\mu}+h_{\mu \nu} x^{\nu}\right.$, with constant $\left.h_{\mu \nu}\right)$, the generators of which will be called $X_{h^{\mu \nu}}$, apply the GAQ formalism and impose the "constraint" $h^{\mu \nu}=h^{\mu \nu}\left(\vec{x}, x^{0}\right)$, on the symplectic submanifolds (solution manifolds). However, the co-homological structure of this finite-dimensional electro-gravitational subgroup, $\tilde{P}_{E G}$ is richer than that of $\tilde{P}_{E}$ and the exponentiation of the Lie algebra $\tilde{\mathcal{P}}_{E G}$ is by far more involved. As mentioned in the Introduction, we attempt only a basic description of the new phenomenology which results from the present revisited gauge principle, although we seek, apart from the exact Lorentz force, an approximate expression for the geodesic force in terms of the metric $g^{\mu \nu} \equiv \eta^{\mu \nu}+h^{\mu \nu}$. Thus, we shall resort only to what seems to be the basic co-homological (fundamental) constants corresponding to the inertial mass $m$, the electric charge $q$, the gravitational mass $g$ and the mixing vertex coupling constant $\kappa$.

Let us write the algebra $\tilde{\mathcal{P}}_{E G}$ in an almost covariant way (the central extensions and induced deformations are necessarily non-covariant). To this end, we parametrize the Lorentz transformations with $\epsilon^{\mu \nu}$ as usual. The proposed explicit algebra is:

$$
\begin{aligned}
{\left[\tilde{X}_{x^{\mu}}^{L}, \tilde{X}_{\epsilon^{\nu \rho}}^{L}\right]=} & -\eta_{\nu \mu} \tilde{X}_{x^{\rho}}^{L}+\eta_{\rho \mu} \tilde{X}_{x^{\nu}}^{L}-(m+\kappa q) c\left(\eta_{\rho \mu} \delta_{\nu}^{0}-\eta_{\nu \mu} \delta_{\rho}^{0}\right) \Xi \\
{\left[\tilde{X}_{x^{\mu}}^{L}, \tilde{X}_{h^{\nu \rho}}^{L}\right]=} & -\eta_{\nu \mu} \tilde{X}_{x^{\rho}}^{L}-\eta_{\rho \mu} \tilde{X}_{x^{\nu}}^{L}+\left[2(g-m c) \eta_{0 \mu} \delta_{\nu}^{0} \delta_{\rho}^{0}+m c\left(\eta_{\rho \mu} \delta_{\nu}^{0}+\eta_{\nu \mu} \delta_{\rho}^{0}\right)\right] \Xi \\
{\left[\tilde{X}_{x^{\mu}}^{L}, \tilde{X}_{A^{\nu}}^{L}\right]=} & -q \eta_{\nu \mu} \Xi \\
{\left[\tilde{X}_{\epsilon^{\mu \nu}}^{L}, \tilde{X}_{\epsilon^{\alpha \beta}}^{L}\right]=} & -\eta_{\alpha \nu} \tilde{X}_{\epsilon^{\mu \beta}}^{L}+\eta_{\beta \nu} \tilde{X}_{\epsilon^{\mu \alpha}}^{L}+\eta_{\alpha \mu} \tilde{X}_{\epsilon^{\nu \beta}}^{L}-\eta_{\mu \beta} \tilde{X}_{\epsilon^{\nu \alpha}}^{L} \\
{\left[\tilde{X}_{\epsilon^{\mu \nu}}^{L}, \tilde{X}_{h^{\alpha \beta}}^{l}\right]=} & -\eta_{\alpha \nu} \tilde{X}_{h^{\mu \beta}}^{L}-\eta_{\beta \nu} \tilde{X}_{h^{\mu \alpha}}^{L}+\eta_{\alpha \mu} \tilde{X}_{h^{\nu \beta}}^{L}+\eta_{\mu \beta} \tilde{X}_{h^{\nu \alpha}}^{L}+ \\
& \frac{1}{q}\left\{\kappa q c\left(\eta_{\alpha \nu} \delta_{\beta}^{\rho} \delta_{\mu}^{0}-\eta_{\mu \alpha} \delta_{\beta}^{\rho} \delta_{\nu}^{0}+\eta_{\nu \beta} \delta_{\alpha}^{\rho} \delta_{\mu}^{0}-\eta_{\mu \beta} \delta_{\alpha}^{\rho} \delta_{\nu}^{0}\right)-\right. \\
& 2(g-m c)\left[\left(\eta_{\alpha \nu} \delta_{\beta}^{\rho} \delta_{\mu}^{0}-\eta_{\mu \alpha} \delta_{\beta}^{\rho} \delta_{\nu}^{0}+\eta_{\nu \beta} \delta_{\alpha}^{\rho} \delta_{\mu}^{0}-\eta_{\mu \beta} \delta_{\alpha}^{\rho} \delta_{\nu}^{0}\right) \delta_{0}^{\rho}+\right. \\
& \left.\left.\delta_{\alpha}^{0} \delta_{\beta}^{0}\left(\eta_{0 \nu} \delta_{\mu}^{0}-\eta_{0 \mu} \delta_{\nu}^{\rho}\right)\right]\right\} \tilde{X}_{A^{\rho}}^{L} \\
{\left[\tilde{X}_{\epsilon^{\mu \nu}}^{L}, \tilde{X}_{A^{\rho}}^{L}\right]=} & -\eta_{\rho \nu} \tilde{X}_{A^{\mu}}^{L}+\eta_{\rho \mu} \tilde{X}_{A^{\nu}}^{L} \\
{\left[\tilde{X}_{h^{\mu \nu}}^{L}, \tilde{X}_{h^{\alpha \beta}}^{L}\right]=} & -\eta_{\alpha \nu} \tilde{X}_{\epsilon^{\mu \beta}}^{L}-\eta_{\beta \nu} \tilde{X}_{\epsilon^{\mu \alpha}}^{L}-\eta_{\alpha \mu} \tilde{X}_{\epsilon^{\nu \beta}}^{L}-\eta_{\mu \beta} \tilde{X}_{\epsilon^{\nu \alpha}}^{L}+ \\
& \frac{1}{q}\left\{-\kappa q c\left[\eta_{\alpha \nu} \delta_{\beta \mu}^{0 \rho}+\eta_{\beta \nu} \delta_{\alpha \mu}^{0 \rho}+\eta_{\alpha \mu} \delta_{\beta \nu}^{0 \rho}+\right.\right. \\
& \left.\left.\eta_{\beta \mu} \delta_{\alpha \nu}^{0 \rho}\right]+2(g-m c)\left[\delta_{\alpha}^{0} \delta_{\beta}^{0}\left(\eta_{0 \nu} \delta_{\mu}^{\rho}+\eta_{0 \beta} \delta_{\nu}^{\rho}\right)-\delta_{\mu}^{0} \delta_{\nu}^{0}\left(\eta_{0 \beta} \delta_{\beta}^{\rho}+\eta_{0 \alpha} \delta_{\beta}^{\rho}\right)\right]\right\} \tilde{X}_{A^{\rho}}^{L} \\
{\left[\tilde{X}_{h^{\mu \nu}}^{L}, \tilde{X}_{A^{\rho}}^{L}\right]=} & -\eta_{\rho \nu} \tilde{X}_{A^{\mu}}^{L}-\eta_{\rho \mu} \tilde{X}_{A^{\nu}}^{L}
\end{aligned}
$$

where $\delta_{\beta \mu}^{0 \rho} \equiv \delta_{\beta}^{0} \delta_{\mu}^{\rho}-\delta_{\mu}^{0} \delta_{\beta}^{\rho}$ is the Kronecker tensor.

It bears mentioning that one of the central extension parameters, actually $g$, is really free at the Lie algebra level but must acquire the value $g=m c$ if the present theory is intended to reproduce the standard disconnected electromagnetic and gravitational forces for $\kappa=0$, i.e. when the constant responsible for the mixing of both interactions is switched off. The appearance of relationships between two co-homology constants, such as $g=m c$, 
indicates the compatibility of further extensions of the algebra $\mathcal{P}_{E G}$ with the constants already introduced. Such further extensions could generalize the present results.

This algebra must be exponentiated in order to have a group law from which to compute left- and right-invariant vector field and the quantization 1-form $\Theta$, just as in the pure electromagnetic example. As stated above, such a process is much more involved and, in principle, a "perturbative" algorithm is in order. We shall resort to an approximation formula [34] up to a given order (order 3 in the fully relativistic case and 4 in the non-relativistic limit given in [14], although in the latter the expressions obtained prove to be exact already at this order), inspired in the theory of formal groups [35], which generalizes that of Campbell-Hausdorff in the sense that it allows for expressions which more directly fit actual physical formulae (although the latter is as well valid). In fact, it has been used in a parallel calculation carried out with REDUCE. Here is the approximate group law:

$$
\begin{aligned}
& x^{\prime \prime \alpha}=x^{\alpha}+x^{\alpha}+\eta_{\mu[\nu} \delta_{\rho]}^{\alpha} \epsilon^{\prime \nu \rho} x^{\mu}+\eta_{\mu(\nu} \delta_{\rho)}^{\alpha} h^{\prime \nu \rho} x^{\mu}+\ldots \\
& \epsilon^{\prime \prime \omega \rho}=\epsilon^{\omega \rho}+\epsilon^{\prime \omega \rho}-\frac{1}{4} \eta_{[\alpha[\nu} \delta_{\mu]}^{[\omega} \delta_{\beta]}^{\rho]} \epsilon^{\prime \mu \nu} \epsilon^{\alpha \beta}-\frac{1}{4} \eta_{(\alpha(\nu} \delta_{\mu)}^{[\omega} \delta_{\beta]}^{\rho]} h^{\prime \mu \nu} h^{\alpha \beta}+\ldots \\
& h^{\prime \prime \omega \rho}=h^{\omega \rho}+h^{\prime \omega \rho}-\frac{1}{2} \eta_{(\alpha[\nu} \delta_{\mu]}^{(\omega} \delta_{\beta)}^{\rho)} \epsilon^{\prime \mu \nu} h^{\alpha \beta}+\ldots \\
& A^{\prime \prime \rho}=A^{\rho}+A^{\prime \rho}+\left(\kappa c \eta_{(\alpha[\nu} \delta_{\mu]}^{0} \delta_{\beta)}^{\rho}\right) \epsilon^{\prime \mu \nu} h^{\alpha \beta}+\eta_{\rho[\mu} \delta_{\nu]}^{\alpha} \epsilon^{\prime \mu \nu} A^{\rho}+ \\
& \frac{1}{2}\left(\kappa c \eta_{(\alpha(\nu} \delta_{\mu)}{ }^{[0} \delta_{\beta)}^{\rho]}\right) h^{\prime \mu \nu} h^{\alpha \beta}-\eta_{\rho(\mu} \delta_{\nu)}^{\alpha} h^{\prime \mu \nu} A^{\rho}+\ldots \\
& \varphi^{\prime \prime}=\varphi^{\prime}+\varphi-(m+\kappa q) c \eta_{\mu[\nu} \delta_{\rho]}{ }^{0} \epsilon^{\prime \mu \nu} x^{\rho}-m c \eta_{\mu(\nu} \delta_{\rho)}{ }^{0} h^{\prime \nu \rho} x^{\mu}+q \eta_{\nu \mu} A^{\prime \nu} x^{\mu}+ \\
& \frac{1}{2}\left\{\left(\frac{-1}{4}(m+\kappa q) c \eta_{\rho[\sigma} \delta_{\gamma]}^{0} \eta_{[\alpha[\nu} \delta_{\mu]}^{[\sigma} \delta_{\beta]}^{\gamma]}-(m+\kappa q) c \eta_{\sigma[\mu} \delta_{\nu]}^{0} \eta_{\rho[\alpha} \delta_{\beta]}^{\sigma}\right)\right) \epsilon^{\prime \mu \nu} \epsilon^{\prime \alpha \beta} x^{\rho}- \\
& m c \eta_{\sigma(\mu} \delta_{\nu)}^{\sigma} \eta_{\rho[\alpha} \delta_{\beta]}^{\sigma} h^{\prime \mu \nu} \epsilon^{\prime \alpha \beta} x^{\rho}+q \eta_{\sigma \mu} \eta_{\rho[\alpha} \delta_{\beta]}^{\sigma} A^{\prime \mu} \epsilon^{\prime \alpha \beta} x^{\rho}+ \\
& {\left[\frac{-1}{4}(m+\kappa q) c \eta_{\rho[\sigma} \delta_{\gamma]}^{0} \eta_{(\alpha(\nu} \delta_{\mu)}^{[\sigma} \delta_{\beta)}^{\gamma]}-\frac{1}{2} \eta_{\rho \sigma}\left(-\kappa q c \eta_{(\alpha(\nu} \delta_{\mu)}{ }^{[0} \delta_{\beta}^{\sigma]}\right)\right] h^{\prime \mu \nu} h^{\prime \alpha \beta} x^{\rho}+} \\
& \left.q \eta_{\sigma \mu} \eta_{\rho(\alpha} \delta_{\beta}{ }^{\sigma} A^{\prime \mu} h^{\alpha \beta} x^{\rho}\right\}+\ldots
\end{aligned}
$$

¿From this law we can proceed following identical steps as in the pure electromagnetic case and derive the approximate quantization form $\Theta$, approximate Noether invariants, Poincaré-Cartan form, Lagrangian, etc.. Let us write explicitly $\Theta$,

$$
\begin{aligned}
\Theta= & d \varphi+ \\
& \left\{(m+\kappa q) c \eta_{\rho[\alpha} \delta_{\beta]}{ }^{0} \epsilon^{\alpha \beta}+m c \eta_{\rho(\alpha} \delta_{\beta}{ }^{0} h^{\alpha \beta}-\right. \\
& q \eta_{\alpha \rho} A^{\alpha}+\frac{1}{4}(m+\kappa q) c\left(\eta_{\sigma[\mu} \delta_{\nu]}{ }^{0} \eta_{\rho[\beta} \delta_{\alpha]}{ }^{\sigma}+\eta_{\sigma[\alpha} \delta_{\beta]}{ }^{0} \eta_{\rho[\nu} \delta_{\mu]}{ }^{\sigma}\right) \epsilon^{\mu \nu} \epsilon^{\alpha \beta}- \\
& \frac{1}{4} m c\left(\eta_{\sigma(\mu} \delta_{\nu)}{ }^{0} \eta_{\rho(\beta} \delta_{\alpha)}^{\sigma}+\eta_{\sigma(\alpha} \delta_{\beta}{ }^{0} \eta_{\rho(\nu} \delta_{\mu)}{ }^{\sigma}\right) h^{\mu \nu} h^{\alpha \beta}+ \\
& \frac{1}{2}\left[2(m+\kappa q) c \eta_{\rho(\mu} \eta_{\nu)[\alpha} \delta_{\beta]}^{0}+m c \eta_{\rho[\alpha} \eta_{\beta](\mu} \delta_{\nu}{ }^{0}\right]+ \\
& \left.\frac{q}{2} \eta_{\nu \rho} \eta_{\omega[\alpha} \delta_{\beta]}{ }^{\nu} \epsilon^{\alpha \beta} A^{\omega}-\frac{q}{2} \eta_{\nu \rho} \eta_{\omega(\alpha} \delta_{\beta)}{ }^{\nu} h^{\alpha \beta} A^{\omega}\right\} d x^{\rho}+\ldots
\end{aligned}
$$


and, before writing $d \Theta$, perform a change of variables in order to take this presymplectic 2-form to almost "canonical" (or standard) form [36]:

$$
\begin{aligned}
A^{\alpha} & \rightarrow A^{\alpha}+\eta_{\sigma \gamma}\left(\epsilon^{\alpha \sigma}+h^{\alpha \sigma}\right) A^{\gamma} \ldots \\
(m+\kappa q) c \epsilon^{0 i} & \rightarrow(m+\kappa q) c \epsilon^{0 i}+\frac{1}{2}(m+\kappa q) c\left(\epsilon^{i j}-\epsilon^{j i}\right) \epsilon^{0 j}+g \delta_{i j} h^{00} \epsilon^{0 j}-2(m+\kappa q) c h^{i j} \epsilon^{0 j}+\ldots \\
h^{0 j} & \rightarrow h^{0 j}+\frac{1}{2}\left(\epsilon^{i j}-\epsilon^{j i}\right) h^{0 i} \\
h^{00} & \rightarrow h^{00}-\frac{1}{4} \delta_{i j} h^{0 i} \epsilon^{0 j}
\end{aligned}
$$

After this change $d \Theta$ acquires the expression:

$$
\begin{aligned}
d \Theta= & d \epsilon^{0 i} \wedge d x^{0}\left(-(m+\kappa q) c\left(\epsilon^{0 i}+h^{0 i}\right)\right)+ \\
& (m+\kappa q) c d \epsilon^{0 i} \wedge d x^{i}+ \\
& d h^{00} \wedge d x^{0} 2 m c\left(1-2 h^{00}\right)+ \\
& d h^{0 i} \wedge d x^{0}\left(m c h^{0 i}-(m+\kappa q) c \epsilon^{0 i}\right)+ \\
& d h^{00} \wedge d x^{i} m c h^{0 i}+ \\
& d h^{0 j} \wedge d x^{i} m c\left(\left(-1+h^{00}\right) \delta_{i j}-h^{j i}\right)- \\
& m c d h^{i j} \wedge d x^{i} h^{0 j}- \\
& q d A^{0} \wedge d x^{0}+ \\
& q d A^{i} \wedge d x^{i}+\ldots
\end{aligned}
$$

The equations of motion can now be obtained á la Cartan by finding the kernel of this Poincaré-Cartan-like form. Then, we have:

$$
\begin{aligned}
(m+\kappa q) c \frac{d^{2} \vec{x}}{d t^{2}}= & q\left[\frac{d \vec{x}}{d t} \wedge \vec{\nabla} \wedge \vec{A}-\partial_{0} \vec{A}-\vec{\nabla} A^{0}\right]+ \\
& m c\left[\partial_{0} \vec{h}+\vec{\nabla} h^{00}-\frac{d \vec{x}}{d t} \wedge \vec{\nabla} \wedge \vec{h}+\right. \\
& \frac{1}{4}\left\{-\partial_{0}\left(h^{00} \vec{h}\right)+\frac{d \vec{x}}{d t} \wedge \vec{\nabla} \wedge\left(h^{00} \vec{h}\right)+\partial_{0}(\overrightarrow{\vec{h}} \cdot \vec{h})-\frac{d \vec{x}}{d t} \wedge \vec{\nabla} \wedge(\overrightarrow{\vec{h}} \cdot \vec{h})-\right. \\
& \left.\left.2 \vec{\nabla}\left(h^{00^{2}}\right)+\vec{\nabla}(\vec{h} \cdot \vec{h})\right\}+\ldots\right]+ \\
& \frac{\kappa q c}{2}\left[\frac{1}{4} \vec{\nabla}(\vec{h} \cdot \vec{h})+\partial_{0} \vec{h}-\frac{d \vec{x}}{d t} \wedge \vec{\nabla} \wedge \vec{h}+\ldots\right]
\end{aligned}
$$

The first line in (52) corresponds to the standard (exact) motion of a particle in the presence of an electromagnetic field, except for the value of the inertial mass, which is corrected by $\kappa q$. The second one reproduces the standard gravito-electromagnetic force [37, i.e. the approximation in which the gravitational field looks like an electromagnetic one. The third and fourth are the first non-linear corrections to gravity. The fifth, however, is quite new and represents a force that looks also like the Lorentz force, at the 
present approximation, but generated by the gravitational potentials, although proportional to $q$; it should not be confused with the above-mentioned gravito-electromagnetic one. As far as the magnitude of the new Lie algebra co-homology constant $\kappa$ is concerned, it is limited by experimental clearance for the difference between particle and anti-particle mass, which for the electron is about $10^{-8} m_{e}$. Even though this is a small value, extremely dense rotating bodies could be able to produce measurable forces. Conversely, a mixing of electromagnetism and gravity predicts a mass difference between charged particles and anti-particles, which could be experimentally tested by measuring, for instance, the Rydberg constant $\left(\sim \frac{m_{\text {antiproton }} m_{\text {positron }}}{m_{\text {antiproton }}+m_{\text {positron }}}\right)$ through the Lamb shift in anti-hydrogen [38].

Let us remark the above-mentioned mentioned fact that one of the allowed Lie-algebra co-homology extension parameters, that is $g$, has been fixed to the particular value $g=m c$ in order to recover the standard theory for $\kappa=0$. We might say that this requirement, along with another condition of "analyticity in $q$ " in the group law, constitutes a grouptheoretical setting of the (weak) Equivalence Principle.

Since the present theory has been formulated on the basis of our group approach to quantization, the quantum version of it would proceed in a rather straightforward manner. We shall not insist any more on this particle mechanical study while waiting for a wide generalization allowing for field degrees of freedom. In fact, a natural yet highly nonelementary attempt at an extension of the present theory to Quantum Field Theory is in course [15]. A further generalization of the present work in which the $U(1)$ subgroup of phase invariance is considered as a Cartan subgroup of a larger internal symmetry group is also in order. Notice that including the phase invariance in, for instance $S U(2) \otimes U(1)$, would result in additional phenomenology such as the production of $Z_{0}$ particles out of gravity.

\section{Acknowledgments}

We desire to thank G. Gibbons for pointing out a relevant misprint in the statement of the no-go theorems in a preliminary version of this letter. We also thank C. Barceló for valuable comments on the Equivalence Principle and S. Vinitsky for exciting comments on the experimental test for the new constant $\kappa$.

\section{References}

[1] R. Hermann, Vector Bundles in Mathematical Physics, N.Y. (1970); Geometry, Physics and Systems, N.Y. (1973).

[2] W. Drechsler and M.E. Mayer, Fibre bundle techniques in gauge theories, Lecture Notes in Physics, 67, Springer-Verlag, Berlin (1977).

[3] R. Utiyama, Phys. Rev. 101, 1597 (1956).

[4] T.W.B. Kibble, J. Math. Phys. 2, 212 (1961). 
[5] L. O’Raifeartaigh, Phys. Rev. B139, 1052 (1965).

[6] S. Coleman and J. Mandula, Phys. Rev. 159, 1251 (1967).

[7] L. Michel, "Invariance in Quantum Mechanics and group extension", in Group theoretical concepts and methods in elementary particle physics, F. Gürsey ed., Gordon \& Breach, pp. 135-200 (1964).

[8] F.J. Dyson, Symmetry Groups in Nuclear and Particle Physics, Benjamin N.Y. (1966).

[9] A. Salam and J. Strathdee, Fortschr. der Phys. 26, 57 (1978).

[10] E. J. Saletan, J. Math. Phys. 2, 1 (1961).

[11] V. Aldaya and J.A. de Azcárraga, Int. J. Teor. Phys. 24, 141 (1985).

[12] V. Aldaya, J. Bisquert, J. Guerrero and J. Navarro-Salas, J. Phys. A26, 5375 (1993).

[13] V. Aldaya, M. Calixto and J.M. Cerveró, Commun. Math. Phys. 200, 325 (1999).

[14] V. Aldaya, J.L. Jaramillo and J. Guerrero, J. Phys. A35, L1 (2002).

[15] V. Aldaya and J.L. Jaramillo, (in preparation).

[16] A.A. Kirillov, Elements of the Theory of Representations, Springer-Verlag (1976).

[17] V. Aldaya and J. de Azcárraga, J. Math. Phys. 23, 1297 (1982).

[18] V. Aldaya, J. Navarro-Salas and A. Ramírez, Commun. Math. Phys., 121, 541 (1989).

[19] J.M. Souriau, Structure des systemes dynamiques, Dunod, Paris (1970).

[20] P. Malliavin, Géometrie différentialle intrinseque, Paris (1972).

[21] C.Godbillon, Géometrie différentielle et mécanique analytique, Hermann, Paris (1969).

[22] R. Abraham and J. E. Marsden, Foundations of Mechanics, W. A. Benjamin, Inc., Reading, Massachusetts, (1967).

[23] J.E. Marsden and T.S. Ratiu, Introduction to Mechanics and Symmetry, SpringerVerlag (1994).

[24] P. Roman, Advanced Quantum Theory, Addison-Wesley (1965).

[25] V. Bargmann, Ann. Math. 48, 568 (1947).

[26] The particular Hamiltonian vector field $X_{H}$ should not be confused with $X^{H}$ defined previously (10). 
[27] We should remark that the symplectic correspondence between functions and (Hamiltonian) vector fields can be kept at the movement-space level provided that we restrict ourselves to only those functions which are invariant under the action of $X^{H}$, i.e. Noether invariants.

[28] B. Kostant, Quantization and Unitary Representations, in Lecture Notes in Math. 170, Springer-Verlag, Berlin (1970).

[29] J. Sniatycki, Geometric Quantization and Quantum Mechanics, Springer-Verlag, New York (1970).

[30] N. Woodhouse, Geometric Quantization, Clarendon, Oxford (1980).

[31] S.Kobayashi and K. Nomizu, Foundations of Differential Geometry Vol. 1, J. Wiley, N.Y. (1996).

[32] In general, $\left[f \otimes X_{a}, g \otimes X_{b}\right]=\left(f L_{X_{a}} g\right) \otimes X_{b}-\left(g L_{X_{b}} f\right) \otimes X_{a}+(f g) \otimes\left[X_{a}, X_{b}\right]$.

[33] V. Aldaya and J.A. de Azcárraga, Int. J. of Theor. Phys. 22, (1983).

[34] V. Aldaya and J. Navarro-Salas, Commun. Math. Phys. 113, 375 (1987).

[35] J.P. Serre Lie Algebras and Lie Groups, W.A. Benjamin, INC., New York, (1965).

[36] This change might have been made in the group law by choicing a more appropriate Campbell-Hausdorff-like formula but that choice is by no means a trivial task. We must stress on the fact that the quantum theory according to GAQ is free of these requirements as we associate quantum operators directly with group generators and the final output. i.e., the quantum representation is fully independent of any choice of co-ordinates in the group manifold. Even more, the classical theory can also be formulated in a fully invariant manner by using the Noether invariants as co-ordinates, but in this way the classical evolution is apparently hidden.

[37] R.M. Wald, General Relativity, The University of Chicago Press (1984).

[38] For a review, see M. Charlton, J. Eades, D. Horvath, R.J. Hughes, C. Zimmermann, Physics Reports 241, 65 (1994). See also G. Baur et al., Phys. Lett. B368, 251 (1996); G. Blanford et al., Phys. Rev. Lett. 80, 3037 (1998). 